\title{
A Competing Risk Nomogram for Predicting Cancer- Specific Survival Among Patients with Prostate Cancer after Radical Prostatectomy
}

\section{Xianghong Zhou}

Sichuan University West China Hospital Department of Urology

Shi Qiu

Sichuan University West China Hospital Department of Urology

Di Jin

Sichuan University West China Hospital Department of Urology

Kun Jin

Sichuan University West China Hospital Department of Urology

Xiaonan Zheng

Sichuan University West China Hospital Department of Urology

Jiakun Li

Sichuan University West China Hospital Department of Urology

\section{Lu Yang}

Sichuan University West China Hospital Department of Urology

Qiang Wei ( $\nabla$ weiqiang933@126.com )

Institute of Urology, West China Hospital of Sichuan University

\section{Research article}

Keywords: Competing risk analyses, Nomogram, Prostate cancer, Radical prostatectomy

Posted Date: October 8th, 2020

DOI: https://doi.org/10.21203/rs.3.rs-86626/v1

License: (c) (1) This work is licensed under a Creative Commons Attribution 4.0 International License. Read Full License 


\section{Abstract}

Background: We aimed to develop a detailed individual survival prognostication tool based on competing risk analyses to predict the risk of 5-year cancer-specific death after radical prostatectomy for patients with prostate cancer (PCa).

Methods: We obtained the data from the Surveillance, Epidemiology, and End Results (SEER) database (2004-2016). The main variables obtained included age at diagnosis, marital status, race, pathological extension, regional lymphonode status, prostate specific antigen level, Gleason Score biopsy. In order to reveal the independent prognostic factors. The cumulative incidence function was used as the univariable competing risk analyses and The Fine and Gray's proportional subdistribution hazard approach was used as the multivariable competing risk analyses. With these factors, a nomogram and risk stratification based on the nomogram was established. Concordance index (C-index) and calibration curves were used for validation.

Results: A total of 95,812 patients were included and divided into training cohort $(n=67,072)$ and validation cohort $(n=28,740)$. Seven independent prognostic factors including age, race, marital status, pathological extension, regional lymphonode status, PSA level, and GS biopsy were used to construct the nomogram. In the training cohort, the C-index was 0.828 (\%95Cl, $0.812-0.844)$, and the $\mathrm{C}$-index was 0.838 (\%95Cl, 0.813-0.863) in the validation cohort. The results of the cumulative incidence function showed that the discrimination of risk stratification based on nomogram is better than that of the risk stratification system based on D'Amico risk stratification.

Conclusions: We successfully developed the first competing risk nomogram to predict the risk of cancerspecific death after surgery for patients with PCa. It has the potential to help clinicians improve postoperative management of patients

\section{Background}

Prostate cancer (PCa) is one of the most common genitourinary tumors. In 2020, it is estimated to cause 33,330 deaths in the United States [1]. Radical prostatectomy (RP) has been confirmed as an effective primary treatment for patients with localized PCa [2]. However, although with the advancement of surgical techniques, a large number of patients undergoing RP have obtained survival benefits, there are still about $25 \%$ of patients who will develop to biochemical recurrence, distant metastasis or even death caused by $\mathrm{PCa}$ [3-6]. At present, it is still controversial as to which kind of patients need to receive active postoperative adjuvant treatment or receive conservative watchful waiting [7]. It is important to identify the patients with a higher risk of recurrence or death, and they may benefit more from postoperative adjuvant treatments.

Some research teams have developed tools to stratify the risk of recurrence or death for PCa patients. For example, D' Amico risk stratification, CAPRA Scoring System and Stephenson nomogram are commonly used in clinical [8-10]. These tools mainly used several clinicopathological parameters such as prostate 
specific antigen (PSA) level, clinical stage, Gleason Score (GS), and pathologic extent to predict the prognosis. However, these tools are still flawed. They are mainly developed based on a small number of patients, the weight between the various prognostic factors is not clear enough and some studies have pointed out that their prediction accuracy is often less than $70 \%[11,12]$. In addition, many patients with $\mathrm{PCa}$ are elderly people with many comorbidities, and they are more likely to die from cardiovascular disease, infection, or other non-tumor factors. Therefore, it is more difficult for researchers to accurately determine the prognosis of patients. $[13,14]$

Competitive risk analysis is a time-to-event analysis, which considers various fatal or non-fatal events that may change or prevent subjects from experiencing interest endpoints such as cancer-specific death, and can more accurately and unbiasedly estimate the patient's true prognosis[14-16]. Meanwhile, the nomogram is a kind of widely used risk prediction tool that can visualize complex regression equations and can analyze the relative weight of each prognostic factor, making the results of the prediction model easier to read and easier to evaluate [17].

To circumvent these defects, with the approach of competing risk analyses, we evaluated the factors affecting prostate cancer-specific survival (CSS) at a large cohort. Furtherly we developed a prognosis nomogram and construct a risk stratification which may have potential clinical implication to help clinicians identify the patients with a high risk of cancer-specific death after RP.

\section{Methods}

\section{Patient selection}

All patients' information was obtained from The Surveillance, Epidemiology, and End Results (SEER) database (2004-2016). SEER database is a public cancer dataset made up of 18 population-based cancer registries. It has covered about $25 \%$ population of the United States. [18] From the SEER database, patients with a diagnosis of adenocarcinoma of the prostate (International Classification of diseases-0-3 code: C61.9) between 2004 and 2016 were selected. Inclusion and exclusion criteria were shown in the flowchart in detail (Fig. 1). With a ratio of 7:3, all the patients were randomly divided into the training cohort and validation cohort.

\section{Variables and endpoint}

For each patient, the information extracted from the SEER database included age at diagnosis, marital status, race, pathological extension, regional lymphonode status, PSA level, GS biopsy, and follow-up information. For continuous variables including age at diagnosis and PSA level, $\mathrm{X}$-tile software (Yale University, USA) was used to assess the optimal cut-off values by the minimal p-value approach [19] (Fig. 2). The optimal cut-off values for age at diagnosis were $\leq 5.9,6.0-14.9,>14$.9. The optimal cut-off values for PSA level were $\leq 5.9,6.0-14.9,>14.9$. 
Cancer-specific death (CSD) was used as the primary endpoint. CSD was measured by all deaths caused by prostate cancer, complications of treatments, or unknown processes in patients with active tumor. Other cause-specific death (OCSD) was measured by all deaths caused by non-cancer events and seen as the competing event of the CSD. Follow-up time was defined as the time between the first treatment and the patient's death or last follow-up.

\section{Statistical analysis}

For categorical variables, a $\mathrm{X}^{2}$ test was used to evaluate the difference between the training cohort and validation cohort, and the results were presented as the frequency with its proportion. In the training cohort, we estimated the cumulative incidence function (CIF) for CSD and tested the survival differences by Gray's test to discover potential prognostic variables with a p-value $<0.05$. Subsequently, we performed competing risk multivariable analyses based on the Fine and Gray's proportional subdistribution hazard approach to identify these independent prognostic variables with a $p$-value $<0.05$. All the independent prognostic factors were selected to construct a nomogram to predict 5-year CSD probabilities for PCa patients after RP.

To validate the performance of the nomogram, in both two cohorts, the discrimination of the nomogram was assessed by Harrell's concordance index (C-index). The value of $\mathrm{C}$-index ranged from 0.5 to 1 , and a higher $\mathrm{C}$-index means better discrimination for prediction model [20]. Besides, the calibration curve with 1000 resamples of bootstrapping was used to compare the predicted survival outcome with the actual survival outcome. The closer the calibration curve was to the standard curve, the closer the survival outcome predicted by the nomogram was to the actual survival outcome. [21]

In addition, we developed a risk stratification based on the nomogram risk score. The cut-off values of risk scores were determined using X-tile software. Then we compare the discrimination abilities of the risk stratification with the European Association of Urology (EAU) risk stratification based on D' Amico stratification [2].

The statistical software R (version 3.4.3, The R Foundation) was used in the above statistical analyses. A p-value $<0.05$ was considered statistically significant.

\section{Results}

\section{Patient characteristics}

Finally, a total of 95,812 eligible patients were included in this study. Among them, 67,072 patients were assigned to the training cohort, while 28,740 patients were assigned to the validation cohort. Table 1 showed the characteristics of patients in detail. Between the training cohort and validation cohorts, there were no statistically significant differences except for the age at diagnosis. 
Table 1

Descriptive characteristics of 95,812 prostate cancer patients undergoing radical prostatectomy between 2004 and 2016 from the Surveillance Epidemiology and End Results database.

\begin{tabular}{|c|c|c|c|c|c|c|c|}
\hline \multirow[t]{2}{*}{ Variable } & \multicolumn{2}{|c|}{$\begin{array}{l}\text { Primary Cohort } \\
(n=95812)\end{array}$} & \multicolumn{2}{|c|}{$\begin{array}{l}\text { Training Cohort } \\
(n=67072)\end{array}$} & \multicolumn{2}{|c|}{$\begin{array}{l}\text { Validation Cohort } \\
(n=28740)\end{array}$} & \multirow[b]{2}{*}{ p-value } \\
\hline & Number & $\%$ & Number & $\%$ & Number & $\%$ & \\
\hline Age & & & & & & & $<0.001$ \\
\hline$\leq 60$ & 39159 & 40.9 & 26320 & 39.2 & 12839 & 44.7 & \\
\hline $61-69$ & 45207 & 47.2 & 32783 & 48.9 & 12424 & 43.2 & \\
\hline$\geq 70$ & 11446 & 11.9 & 7969 & 11.9 & 3477 & 12.1 & \\
\hline Race $^{1}$ & & & & & & & 0.247 \\
\hline White & 78440 & 81.9 & 54968 & 82.0 & 23472 & 81.7 & \\
\hline Black & 12182 & 12.7 & 8524 & 12.7 & 3658 & 12.7 & \\
\hline Other & 5190 & 5.4 & 3580 & 5.3 & 1610 & 5.6 & \\
\hline Marital Status & & & & & & & 0.110 \\
\hline Married & 77053 & 80.4 & 54030 & 73.4 & 23023 & 73.8 & \\
\hline Single ${ }^{2}$ & 18759 & 19.6 & 13042 & 21.1 & 5717 & 21.2 & \\
\hline Pathological extension & & & & & & & 0.080 \\
\hline Organ-confined & 67469 & 70.4 & 47120 & 70.3 & 20349 & 70.8 & \\
\hline Extracapsule-invasion & 18041 & 18.8 & 12655 & 18.9 & 5386 & 18.7 & \\
\hline Seminal vesicle invasion & 9371 & 9.8 & 6658 & 9.9 & 2713 & 9.4 & \\
\hline Adjacent structures invasion & 931 & 1.0 & 639 & 1.0 & 292 & 1.0 & \\
\hline Regional lymphonode status & & & & & & & 0.346 \\
\hline negetive & 91526 & 95.5 & 64044 & 86.4 & 27482 & 95.6 & \\
\hline positive & 4286 & 4.5 & 3028 & 13.6 & 1258 & 4.4 & \\
\hline PSA level (ng/ml) & & & & & & & 0.777 \\
\hline$\leq 5.9$ & 45664 & 47.7 & 31971 & 47.7 & 13693 & 47.6 & \\
\hline
\end{tabular}

1.Black: African American, White: Caucasian, Other: American Indian/AK Native, Asian/Pacific Islander

2.Single: Divorced, Separated, Single (never married), Widowed, unmarried 


\begin{tabular}{|c|c|c|c|c|c|c|c|}
\hline \multirow[t]{2}{*}{ Variable } & \multicolumn{2}{|c|}{$\begin{array}{l}\text { Primary Cohort } \\
(n=95812)\end{array}$} & \multicolumn{2}{|c|}{$\begin{array}{l}\text { Training Cohort } \\
(n=67072)\end{array}$} & \multicolumn{2}{|c|}{$\begin{array}{l}\text { Validation Cohort } \\
(n=28740)\end{array}$} & \multirow[b]{2}{*}{ p-value } \\
\hline & Number & $\%$ & Number & $\%$ & Number & $\%$ & \\
\hline $6.0-14.9$ & 39882 & 41.6 & 27886 & 41.6 & 11996 & 41.7 & \\
\hline$>14.9$ & 10266 & 10.7 & 7215 & 10.8 & 3051 & 10.6 & \\
\hline \multicolumn{3}{|l|}{ GS biopsy } & & & & & 0.131 \\
\hline$\leq 6$ & 23522 & 24.6 & 16444 & 24.5 & 7078 & 24.6 & \\
\hline $7(3+4)$ & 40563 & 42.3 & 28334 & 42.2 & 12229 & 42.6 & \\
\hline $7(4+3)$ & 16887 & 17.6 & 11809 & 17.6 & 5078 & 17.7 & \\
\hline $7(N / A)$ & 108 & 0.1 & 73 & 0.1 & 35 & 0.1 & \\
\hline 8 & 6908 & 7.2 & 4878 & 7.3 & 2030 & 7.1 & \\
\hline 9 & 7581 & 7.9 & 5379 & 8.0 & 2202 & 7.7 & \\
\hline 10 & 243 & 0.3 & 155 & 0.2 & 88 & 0.3 & \\
\hline \multicolumn{3}{|l|}{ EAU risk classification } & & & & & 0.455 \\
\hline Low risk & 3316 & 3.5 & 2306 & 3.4 & 1010 & 3.5 & \\
\hline Intermediate risk & 5250 & 5.5 & 3654 & 5.4 & 1596 & 5.6 & \\
\hline High risk & 76649 & 80.0 & 53630 & 80.0 & 23019 & 80.1 & \\
\hline Unknown & 10597 & 11.1 & 7482 & 11.2 & 3115 & 10.8 & \\
\hline \multicolumn{8}{|c|}{$\begin{array}{l}\text { 1.Black: African American, White: Caucasian, Other: American Indian/AK Native, Asian/Pacific } \\
\text { Islander }\end{array}$} \\
\hline \multicolumn{7}{|c|}{ 2.Single: Divorced, Separated, Single (never married), Widowed, unmarried } & \\
\hline
\end{tabular}

\section{Identification of independent prognostic factors}

We performed the analyses of CIF and Gray's test as the univariable analyses. The results showed that age, race, marital status, pathological extension, regional lymphonode status, PSA level, and GS biopsy were the factors with a significant impact on CSD. The Fine and Gray's proportional subdistribution hazard approach was performed as the multivariable analyses. And the results were consistent, in which age, race, marital status, pathological extension, regional lymphonode status, PSA level, and GS biopsy were the significant prognostic factors of CSD. These variables could be thought as the independent prognostic factor for predicting the CSS of PCa patients after RP. The detailed results of univariable and multivariable analyses were showed in Table 2. 
Table 2

Univariate analyses and multivariate Competing risk analyses of prognostic factors influencing cancerspecific survival outcomes in the training cohort

\begin{tabular}{|c|c|c|c|}
\hline Variable & $\begin{array}{l}\text { Univariate } \\
\text { analyses } \\
\text { (CIF) } \\
\text { p-value }\end{array}$ & $\begin{array}{l}\text { Multivariate Competing risk } \\
\text { analyses } \\
\text { sdHR }(95 \% \mathrm{Cl})\end{array}$ & p-value \\
\hline Age & $<0.001$ & & \\
\hline$\leq 60$ & & Ref. & \\
\hline $61-69$ & & $1.098(0.972-1.240)$ & 0.132 \\
\hline$\geq 70$ & & $1.308(1.103-1.551)$ & 0.002 \\
\hline Race $^{1}$ & 0.024 & & \\
\hline White & & Ref. & \\
\hline Black & & $1.225(1.043-1.440)$ & 0.014 \\
\hline Other & & $0.698(0.529-0.920)$ & 0.010 \\
\hline Marital Status & $<0.001$ & & \\
\hline \multicolumn{4}{|l|}{ Married } \\
\hline Single ${ }^{2}$ & & $1.253(1.099-1.429)$ & $<0.001$ \\
\hline \multicolumn{4}{|l|}{ Pathological extension } \\
\hline Organ-confined & & Ref. & \\
\hline Extracapsule-invasion & & $2.273(1.956-2.641)$ & $<0.001$ \\
\hline Seminal vesicle invasion & & $3.576(3.039-4.207)$ & $<0.001$ \\
\hline \multirow{2}{*}{$\begin{array}{l}\text { Adjacent structures } \\
\text { invasion }\end{array}$} & & $4.245(3.261-5.525)$ & $<0.001$ \\
\hline & & & $<0.001$ \\
\hline $\begin{array}{l}\text { Regional lymphonode } \\
\text { status }\end{array}$ & $<0.001$ & & \\
\hline negetive & & Ref. & \\
\hline
\end{tabular}

1.Black: African American, White: Caucasian, Other: American Indian/AK Native, Asian/Pacific Islander

2.Single: Divorced, Separated, Single (never married), Widowed, unmarried

Abbreviation: CIF: cumulative incidence function, sdHR: subdistribution hazard ratio, Ref.: reference 


\begin{tabular}{|c|c|c|c|}
\hline Variable & $\begin{array}{l}\text { Univariate } \\
\text { analyses } \\
\text { (CIF) } \\
\text { p-value }\end{array}$ & $\begin{array}{l}\text { Multivariate Competing risk } \\
\text { analyses } \\
\text { sdHR }(95 \% \mathrm{Cl})\end{array}$ & p-value \\
\hline \multicolumn{2}{|l|}{ positive } & $1.917(1.647-2.231)$ & $<0.001$ \\
\hline PSA level (ng/ml) & $<0.001$ & & \\
\hline \multicolumn{2}{|l|}{$\leq 5.9$} & Ref. & \\
\hline \multicolumn{2}{|l|}{$6.0-14.9$} & $1,252(1.043-1.440)$ & $<0.001$ \\
\hline \multicolumn{2}{|l|}{$>14.9$} & $1.430(1.214-1.686)$ & $<0.001$ \\
\hline GS biopsy & $<0.001$ & & \\
\hline \multicolumn{2}{|l|}{$\leq 6$} & Ref. & \\
\hline \multicolumn{2}{|l|}{$7(3+4)$} & $1.647(1.291-2.101)$ & $<0.001$ \\
\hline \multicolumn{2}{|l|}{$7(4+3)$} & $3.150(2.441-4.065)$ & $<0.001$ \\
\hline \multicolumn{2}{|l|}{$7(\mathrm{~N} / \mathrm{A})$} & $3.980(1.253-12.641)$ & 0.019 \\
\hline \multicolumn{2}{|l|}{8} & $5.065(3.900-6.583)$ & $<0.001$ \\
\hline \multicolumn{2}{|l|}{9} & $10.827(8.448-13.874)$ & $<0.001$ \\
\hline \multicolumn{2}{|l|}{10} & $20.528(13.803-30.978)$ & $<0.001$ \\
\hline \multicolumn{4}{|c|}{$\begin{array}{l}\text { 1.Black: African American, White: Caucasian, Other: American Indian/AK Native, Asian/Pacific } \\
\text { Islander }\end{array}$} \\
\hline \multicolumn{4}{|c|}{ 2.Single: Divorced, Separated, Single (never married), Widowed, unmarried } \\
\hline \multicolumn{4}{|c|}{ Abbreviation: CIF: cumulative incidence function, sdHR: subdistribution hazard ratio, Ref.: reference } \\
\hline
\end{tabular}

\section{Development and validation of the competing risk nomogram}

Based on the results of univariable and multivariable competing risk analyses, age, race, marital status, pathological extension, regional lymphonode status, PSA level, and GS biopsy were used to construct the nomogram for predicting the probability of 5-year CSD for PCa patients after RP (Fig. 3). The detailed score of each nomogram variable was listed in Table 3. 
Table 3

Detailed risk scores of all independent prognostic factors in the nomogram

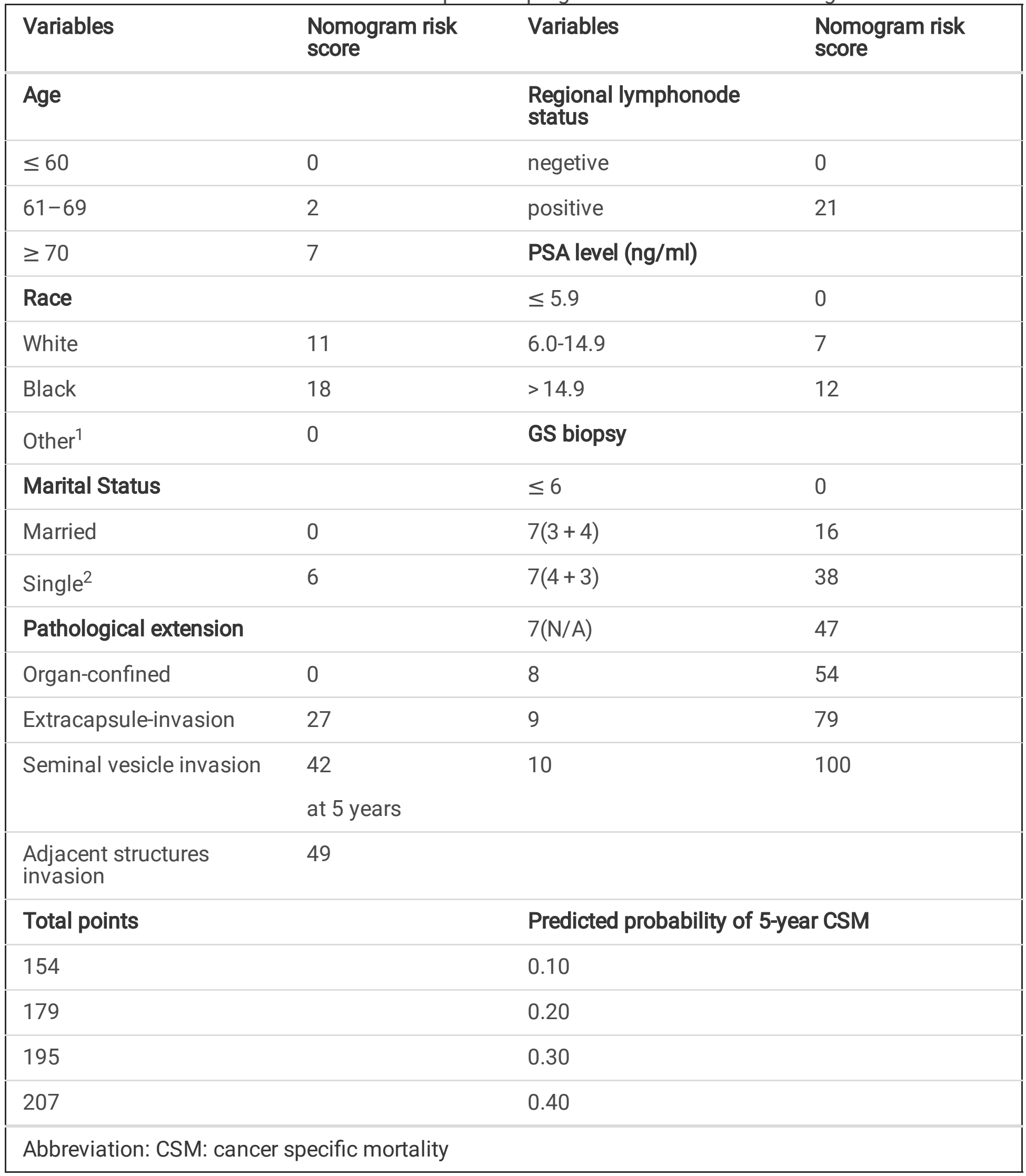

We performed the analyses of C-index and calibration curve to validate the reliability of the nomogram. For the training cohort, the $\mathrm{C}$-index was 0.828 (\%95Cl, 0.812-0.844). For the validation cohort, the C-index was $0.838(\% 95 \mathrm{Cl}, 0.813-0.863)$. The relatively high C-index (>0.8) showed the good predictive ability of 
this nomogram. Meanwhile, in both training cohort and validation cohort, the calibration curves showed a good agreement between the 5-year CSD predicted by nomogram and actual 5-year CSD (Fig. 4).

\section{Establishment of risk stratification for cancer-specific death after RP}

According to the score corresponding to each nomogram variable, we calculated the total risk score for each patient in both the training cohort and the validation cohort. By the X-tile approach, patients were divided into three risk groups based on the total risk score from the nomogram. The low-risk group included patients with 0-66 points, the middle-risk group included patients with 67-105 points, and the high-risk group included patients with no less than 106 points.

In order to verify the predictive value of this risk stratification, we compared it with the EAU risk stratification based on D' Amico stratification. EAU risk stratification is one of the most popular risk stratification tools for PCa patients, which can divide patients into three groups including low-risk, medium-risk, and high-risk. In both training cohort and validation cohort, we plotted the CIF curves for different risk groups based on the risk stratification of the nomogram or EAU risk stratification (Fig. 5). Compared with our risk groups, the degree of separation of CIF curves of CSD between groups was more obvious than EAU risk stratification. Meanwhile, in both two cohorts, The high-risk group identified by our risk stratification had a significantly higher CSD risk than OCSD, while the high-risk group in the EAU risk stratification did not. The results showed that the novel risk stratification based on the nomogram had better prognostic discrimination than EAU risk stratification.

\section{Discussion}

In this study, based on a large cohort of 95812 patients from SEER database, we identified seven risk factors and construct a competing risk nomogram based on these prognostic factors to predict the probability of the occurrence of CSD within 5 years after RP for each patient with PCa. Furthermore, based on the difference in nomogram scores, we developed a novel risk stratification for postoperative CSD in patients with PCa. Our risk stratification has potential clinical value and may help clinicians better identify patients who still need active intervention after RP. The results showed that the discrimination of our stratification system was not weaker than the commonly used EAU risk stratification based on D'Amico stratification.

Competing risk nomogram is a kind of widely used risk predicting model in many fields in oncology such as lung cancer, breast cancer, and colorectal cancer [22-24]. The nomogram can incorporate many key factors of the disease into the prognosis prediction model and can consider the weight of each variable to make the prediction model more accurate. In addition, the graphical representation helps to more intuitively evaluate the individual situation of each patient, which is more practical. [25] At the same time, competing risk nomogram has its unique advantages compared to traditional nomogram or other 
prognosis predicting models. The competitive risk nomogram is based on competing risk analysis methods such as CIF and Fine and Gray's proportional subdistribution hazard approach, rather than the Kaplan-meier method and Cox proportional risk regression commonly used in other types of models [16]. Competitive risk analyses not only consider the survival and death of patients, but also consider the impact of death caused by other factors on the endpoints of interest such as CSD. This is especially important in the research of $\mathrm{PCa}$, because a large part of PCa patients may die due to other factors before developing CSD [13]. To our knowledge, there is still no research reported on the competitive risk prognosis prediction model for the prognosis of PCa patients after RP.

In the field of PCa, the currently commonly used nomogram is Stephenson nomogram. It is developed by Stephenson et al. to predict disease progression after salvage radiotherapy (SRT), with data from a multi-institutional retrospective cohort of 1540 patients. Seven variables were used to construct the nomogram including PSA before SRT, surgical margins, GS, PSA double time before SRT, lymph node metastasis and androgen deprivation therapy administration before or during SRT. [26] However, there are still some defects with Stephenson nomogram. Due to the limitation of inclusion and exclusion criteria, it is not widely applicable to PCa patients who have received RP. At the same time, it paid little attention to hard endpoints such as CSD. In the cohort of the original study, its c-index was 0.69 , and the c-index obtained after the test in another study was even lower [27]. Therefore, for predicting the survival of PCa patients after RP better, a more accurate and versatile nomogram is still needed.

In our study, the competing risk analyses identified 7 prognostic factors including age, race, marital status, pathological extension, regional lymphonode status, PSA level, and GS biopsy. Among them, GS had the greatest influence on survival outcomes. Many studies have reported the relationship between GS and the prognosis of PCa [28-30]. International Society of Urological Pathology (ISUP) reported that GS can be divided into five groups $(2-6,7(3+4), 7(4+3), 8, \geq 9)$ according to prognosis, and this was consistent with our research results [30]. With the increase of GS, the patient's nomogram score was also increasing, that is to say, the possibility of the patient developing CSD within 5 years was increasing. In the nomogram, we could find that GS $4+3=7$ group was with an obviously higher score than GS $3+4=7$ group with. This was also consistent with the latest American Urological Association (AUA) clinical guideline, which indicated that many researches had demonstrated that the prognosis of GS $4+3$ was significantly worse than GS $3+4[31,32]$. Pathological extension was another important prognostic factor whose weight was second only to GS. It has been widely accepted that poor pathological findings such as extracapsular invasion and seminal vesicle invasion are related to disease recurrence and poor prognosis [33-35].

In addition to the above-mentioned well-known prognostic factors, our study also found the impact of race and marital status on the prognosis of PCa patients. Our nomogram showed that African Americans had the highest risk of CSD after RP, followed by Caucasian and other races. This finding was consistent with some studies published in recent years. According to statistics from researchers, the average annual incidence of PCa among African Americans was $60 \%$ higher than that of Caucasian men. Besides, compared with other races, African Americans have the highest mortality rate [36,37]. The causes of the 
result were very complicated. For example, In the United States, PCa tended to be larger in African Americans and was more likely to metastasize than white men [38]. From a genetic perspective, some gene mutations related to disease progression are more common in African Americans, such as TP53 mutations and MYC amplification [39]. Several risk-associated single nucleotide polymorphisms were found to be overexpressed in African Americans [40]. At the same time, African Americans may face some social barriers such as health insurance, which may affect the treatment and management of the disease [41]. Our competing risk analyses also identified marital status as an independent prognostic factor. More and more researchers have paid attention to the impact of this sociological factor on the disease. Outcomes of numerous studies showed that married marital status was a protective factor for the occurrence and development of a variety of tumors, including PCa. Marriage may be a multifaceted representation of many protective factors including social support. [42, 43]

EAU risk stratification based on D' Amico stratification is currently a common risk stratification system for PCa patients, which divided patients into Low-risk group, Intermediate-risk group, and High-risk group for predicting the risk of disease recurrence [2]. In our study, we compared the novel risk stratification based on the nomogram with EAU risk stratification. The results showed that our risk stratification system had better discrimination with a C-index over 0.8 and could better detect patients at higher risk of the occurrence of postoperative CSD after adjustment of competing risk analyses. The high-risk group obtained through our risk stratification had a significantly higher risk of CSD than OCSD, which could better exclude the interference of death caused by non-tumor factors on the model. Our advantages may come from many aspects, such as a large cohort, more prognostic factors, and independent analyses of competing risks. At the same time, our research provides quantitative and graphical prognostic tools, which help to make more accurate assessments of each patient.

Our study revealed 7 main independent prognostic factors that affect the occurrence of CSD in patients after RP and explored the application of these factors in identifying high-risk patients through the nomogram and risk stratification. At present, the guidelines pointed out that there were multiple managements for patients undergoing RP, including adjuvant treatment, salvage treatment, watchful waiting, etc. However, due to the lack of high-quality prospective data, the inclusion and exclusion criteria of patients are still controversial. [2,44] In our study, the risk stratification proposed by the nomogram provided a reference for the selection criteria for the postoperative management of patients. Taking into account the differences in the risk of CSD, the high-risk group may require more active intervention, while the low-risk group may be more suitable for watchful waiting.

There are still several limitations to our study. First, our research is based on a large retrospective cohort. We still need more prospective clinical trials to contribute more precise data. Second, due to the SEER database's limitations, we are unable to obtain some data that can enrich our research outcomes, such as patients' functional status and disease progression, as well as some more detailed clinical parameters such as PSA double time. Although our prediction model has reached a relatively high accuracy (C-index $>0.8$ ), in the future we can try to use these parameters to further optimize the nomogram and risk 
stratification system. Third, we also lack additional independent external validation sets, and this is our important work goal in the future.

\section{Conclusions}

In conclusion, we performed a competing risk analysis based on a larger cohort of 95,812 patients with nonmetastatic PCa from the SEER database. We also identified 7 independent prognostic factors of the occurrence of CSD after RP, and constructed a competing risk nomogram utilizing the 7 factors for detecting the risk of CSD for each patient. A risk stratification system was established based on the nomogram to help clinicians better identify patients at high risk of CSD after surgery.

\section{Abbreviations}

PCa: Prostate cancer; Cl: confidence interval; RP: radical prostatectomy; salvage radiotherapy (SRT); GS: Gleason Score; CSS: cancer-specific survival; CSM: cancer-specific mortality; CIF: cumulative incidence function; PSA: prostate specific antigen; SEER: the Surveillance, Epidemiology, and End Results; CSD: Cancer-specific death; OCSD: Other cause-specific death; EAU: European Association of Urology; ISUP: International Society of Urological Pathology; AUA: American Urological Association

\section{Declarations}

\section{Acknowledgments}

The authors acknowledge the efforts of the Surveillance Research Program in the establishment and maintenance of the SEER database.

\section{Funding}

This work was supported by the National key research and development program of China (Grant No. 2017YFC0908003), National Natural Science Foundation of China (Grant No.81902578, 81974098), China Postdoctoral Science Foundation (2017M612971), Post-doctoral Science Research Foundation of Sichuan University (2020SCU12041), Post-Doctor Research Project, West China Hospital, Sichuan University (2018HXBH085), National Clinical Research Center for Geriatrics, West China Hospital, Sichuan University (Z2018C01) .

\section{Availability of data and materials}

All analyzed data are publicly available at the SEER website (http://www.seer.cancer.gov), and should be requested under the approval of the SEER Program administration. 


\section{Authors' contributions}

$\mathrm{XHZ}, \mathrm{SQ}, \mathrm{LY}$ and $\mathrm{QW}$ are responsible for designing the study, writing, collecting data, analysis, and final approval of the article. DJ, KJ are responsible for a part of analysis and revision. XNZ and JKL are responsible for a part of writing and revision.

All authors have read and approved the final manuscript.

\section{Ethics approval and consent to participate}

This study used atomized patient's data of a public dataset from the Surveillance, Epidemiology, and End Results database, and therefore did not require institutional review board approval.

\section{Consent for publication}

All authors agreed to publish the manuscript titled "A Competing Risk Nomogram for Predicting CancerSpecific Survival Among Patients with Prostate Cancer after Radical Prostatectomy" in the journal BMC Cancer.

\section{Competing interests}

The authors declare that they have no competing interests.

\section{References}

1. Siegel, R.L., K.D. Miller and A. Jemal.Cancer statistics, 2020.CA Cancer J Clin 2020; 70: 7-30.

2. Mottet, N., J. Bellmunt, M. Bolla, E. Briers, M.G. Cumberbatch, and M. De Santis, et al.EAU-ESTROSIOG Guidelines on Prostate Cancer. Part 1: Screening, Diagnosis, and Local Treatment with Curative Intent.Eur Urol 2017; 71: 618-629.

3. Cornford, P., J. Bellmunt, M. Bolla, E. Briers, M. De Santis, and T. Gross, et al.EAU-ESTRO-SIOG Guidelines on Prostate Cancer. Part II: Treatment of Relapsing, Metastatic, and Castration-Resistant Prostate Cancer.Eur Urol 2017; 71: 630-642.

4. Mikel, H.J., S.A. Boorjian, I. Frank, M.T. Gettman, T.R. Houston, and L.J. Rangel, et al.The presence of extracapsular extension is associated with an increased risk of death from prostate cancer after radical prostatectomy for patients with seminal vesicle invasion and negative lymph nodes.Urol Oncol 2014; 32: 26.e1-7.

5. Abdollah, F., S. Boorjian, C. Cozzarini, N. Suardi, M. Sun, and C. Fiorino, et al.Survival following biochemical recurrence after radical prostatectomy and adjuvant radiotherapy in patients with 
prostate cancer: the impact of competing causes of mortality and patient stratification.Eur Urol 2013; 64: 557-64.

6. Zhang, T., A.J. Armstrong, D.J. George, and J. Huang.The promise of immunotherapy in genitourinary malignancies.Precis Clin Med 2018; 1: 97-101.

7. Patel, A.R. and A.J. Stephenson.Radiation therapy for prostate cancer after prostatectomy: adjuvant or salvage? Nat Rev Urol 2011; 8: 385-92.

8. D'Amico, A.V., R. Whittington, S.B. Malkowicz, D. Schultz, K. Blank, and G.A. Broderick, et al.Biochemical outcome after radical prostatectomy, external beam radiation therapy, or interstitial radiation therapy for clinically localized prostate cancer.JAMA 1998; 280: 969-74.

9. Cooperberg, M.R., D.J. Pasta, E.P. Elkin, M.S. Litwin, D.M. Latini, and J. Du Chane, et al.The University of California, San Francisco Cancer of the Prostate Risk Assessment score: a straightforward and reliable preoperative predictor of disease recurrence after radical prostatectomy.J Urol 2005; 173 : 1938-42.

10. Stephenson, A.J., P.T. Scardino, J.A. Eastham, F.J. Bianco, Z.A. Dotan, and P.A. Fearn, et al.Preoperative nomogram predicting the 10-year probability of prostate cancer recurrence after radical prostatectomy.J Natl Cancer Inst 2006; 98: 715-7.

11. Eggener, S.E., P.T. Scardino, P.C. Walsh, M. Han, A.W. Partin, and B.J. Trock, et al.Predicting 15-year prostate cancer specific mortality after radical prostatectomy.J Urol 2011; 185: 869-75.

12. Zhang, Y.D., C.J. Wu, M.L. Bao, H. Li, X.N. Wang, and X.S. Liu, et al.MR-based prognostic nomogram for prostate cancer after radical prostatectomy.J Magn Reson Imaging 2017; 45: 586-596.

13. Vagnoni, V., L. Bianchi, M. Borghesi, C.V. Pultrone, H. Dababneh, and F. Chessa, et al.Adverse Features and Competing Risk Mortality in Patients With High-Risk Prostate Cancer.Clin Genitourin Cancer 2017; 15: e239-e248.

14. Zhou, X., Q. Ning, K. Jin, T. Zhang, and X. Ma.Development and validation of a preoperative nomogram for predicting survival of patients with locally advanced prostate cancer after radical prostatectomy.BMC Cancer 2020; 20: 97.

15. Gooley, T.A., W. Leisenring, J. Crowley, and B.E. Storer.Estimation of failure probabilities in the presence of competing risks: new representations of old estimators. Stat Med 1999; 18: 695-706.

16. Austin, P.C., D.S. Lee and J.P. Fine.Introduction to the Analysis of Survival Data in the Presence of Competing Risks.Circulation 2016; 133: 601-9.

17. Shen, W., N. Sakamoto and L. Yang.Model to Predict Cause-Specific Mortality in Patients with Head and Neck Adenoid Cystic Carcinoma: A Competing Risk Analysis.Ann Surg Oncol 2017; 24: 21292136.

18. Cronin, K.A., L.A. Ries and B.K. Edwards. The Surveillance, Epidemiology, and End Results (SEER) Program of the National Cancer Institute.Cancer 2014; 120 Suppl 23: 3755-7.

19. Camp, R.L., M. Dolled-Filhart and D.L. Rimm.X-tile: a new bio-informatics tool for biomarker assessment and outcome-based cut-point optimization.Clin Cancer Res 2004; 10: 7252-9. 
20. Weiss, A., M. Chavez-MacGregor, D.Y. Lichtensztajn, M. Yi, A. Tadros, and G.N. Hortobagyi, et al.Validation Study of the American Joint Committee on Cancer Eighth Edition Prognostic Stage Compared With the Anatomic Stage in Breast Cancer.JAMA Oncol 2018; 4: 203-209.

21. Van Calster, B., D.J. McLernon, M. van Smeden, L. Wynants, and E.W. Steyerberg.Calibration: the Achilles heel of predictive analytics.BMC Med 2019; 17: 230.

22. Zhou, H., J. Shen, Y. Zhang, Y. Huang, W. Fang, and Y. Yang, et al.Risk of second primary malignancy after non-small cell lung cancer: a competing risk nomogram based on the SEER database.Ann Transl Med 2019; 7: 439.

23. Shen, K., L. Yao, J. Wei, Z. Luo, W. Yu, and H. Zhai, et al.Worse characteristics can predict survival effectively in bilateral primary breast cancer: A competing risk nomogram using the SEER database.Cancer Med 2019; 8: 7890-7902.

24. Song, W. and T. Fu.Circular RNA-Associated Competing Endogenous RNA Network and Prognostic Nomogram for Patients With Colorectal Cancer.Front Oncol 2019; 9: 1181.

25. Balachandran, V.P., M. Gonen, J.J. Smith, and R.P. DeMatteo.Nomograms in oncology: more than meets the eye.Lancet Oncol 2015; 16: e173-80.

26. Stephenson, A.J., P.T. Scardino, M.W. Kattan, T.M. Pisansky, K.M. Slawin, and E.A. Klein, et al.Predicting the outcome of salvage radiation therapy for recurrent prostate cancer after radical prostatectomy.J Clin Oncol 2007; 25: 2035-41.

27. Moreira, D.M., J. Jayachandran, J.J. Presti, W.J. Aronson, M.K. Terris, and C.J. Kane, et al.Validation of a nomogram to predict disease progression following salvage radiotherapy after radical prostatectomy: results from the SEARCH database.BJU Int 2009; 104: 1452-6.

28. Sundi, D., V. Wang, P.M. Pierorazio, M. Han, A.W. Partin, and P.T. Tran, et al.Identification of men with the highest risk of early disease recurrence after radical prostatectomy.Prostate 2014; 74: 628-36.

29. Pierorazio, P.M., P.C. Walsh, A.W. Partin, and J.I. Epstein.Prognostic Gleason grade grouping: data based on the modified Gleason scoring system.BJU Int 2013; 111: 753-60.

30. Epstein, J.I., L. Egevad, M.B. Amin, B. Delahunt, J.R. Srigley, and P.A. Humphrey.The 2014 International Society of Urological Pathology (ISUP) Consensus Conference on Gleason Grading of Prostatic Carcinoma: Definition of Grading Patterns and Proposal for a New Grading System.Am J Surg Pathol 2016; 40: 244-52.

31. Sanda, M.G., J.A. Cadeddu, E. Kirkby, R.C. Chen, T. Crispino, and J. Fontanarosa, et al.Clinically Localized Prostate Cancer: AUA/ASTRO/SUO Guideline. Part II: Recommended Approaches and Details of Specific Care Options.J Urol 2018; 199: 990-997.

32. Mathieu, R., M. Moschini, B. Beyer, K.M. Gust, T. Seisen, and A. Briganti, et al.Prognostic value of the new Grade Groups in Prostate Cancer: a multi-institutional European validation study.Prostate Cancer Prostatic Dis 2017; 20: 197-202.

33. Forgues, A., F. Rozet, F. Audenet, A. Ouzzane, R. Sanchez-Salas, and E. Barret, et al.Oncologic outcomes after minimally invasive radical prostatectomy in patients with seminal vesicle invasion (pT3b) without adjuvant therapy.World J Urol 2014; 32: 519-24. 
34. Epstein, J.I., A.W. Partin, S.R. Potter, and P.C. Walsh.Adenocarcinoma of the prostate invading the seminal vesicle: prognostic stratification based on pathologic parameters.Urology 2000; 56: 283-8.

35. Grignon, D.J.Prostate cancer reporting and staging: needle biopsy and radical prostatectomy specimens.Mod Pathol 2018; 31: S96-109.

36. Rebbeck, T.R.Prostate Cancer Disparities by Race and Ethnicity: From Nucleotide to Neighborhood.Cold Spring Harb Perspect Med 2018; 8.

37. Odedina, F.T., T.O. Akinremi, F. Chinegwundoh, R. Roberts, D. Yu, and R.R. Reams, et al.Prostate cancer disparities in Black men of African descent: a comparative literature review of prostate cancer burden among Black men in the United States, Caribbean, United Kingdom, and West Africa.Infect Agent Cancer 2009; 4 Suppl 1: S2.

38. Powell, I.J., C.H. Bock, J.J. Ruterbusch, and W. Sakr.Evidence supports a faster growth rate and/or earlier transformation to clinically significant prostate cancer in black than in white American men, and influences racial progression and mortality disparity.J Urol 2010; 183: 1792-6.

39. Koga, Y., H. Song, Z.R. Chalmers, J. Newberg, E. Kim, and J. Carrot-Zhang, et al.Genomic Profiling of Prostate Cancers from Men with African and European Ancestry.Clin Cancer Res 2020.

40. Du XL, S. Fang, A.L. Coker, M. Sanderson, C. Aragaki, and J.N. Cormier, et al.Racial disparity and socioeconomic status in association with survival in older men with local/regional stage prostate carcinoma: findings from a large community-based cohort.Cancer 2006; 106: 1276-85.

41. Fiscella, K. and M.R. Sanders.Racial and Ethnic Disparities in the Quality of Health Care.Annu Rev Public Health 2016; 37: 375-94.

42. Khan, S., K.G. Nepple, A.S. Kibel, G. Sandhu, D. Kallogjeri, and S. Strope, et al.The association of marital status and mortality among men with early-stage prostate cancer treated with radical prostatectomy: insight into post-prostatectomy survival strategies. Cancer Causes Control 2019; 30: 871-876.

43. Aizer, A.A., M.H. Chen, E.P. McCarthy, M.L. Mendu, S. Koo, and T.J. Wilhite, et al.Marital status and survival in patients with cancer.J Clin Oncol 2013; 31: 3869-76.

44. Sanda, M.G., J.A. Cadeddu, E. Kirkby, R.C. Chen, T. Crispino, and J. Fontanarosa, et al.Clinically Localized Prostate Cancer: AUA/ASTRO/SUO Guideline. Part I: Risk Stratification, Shared Decision Making, and Care Options.J Urol 2018; 199: 683-690.

\section{Figures}


Patients diagnosed with prostate cancer between 2004-2016 from SEER database

Histology different from adenocarcinoma or histology unknown or not primary tumor

Patients 1) not confirmed by positive histology or 2 ) confirmed by autopsy or 3 ) accompanied with other kinds of tumor 4) unknown cause of death

Patients with metastatic tumor

Patients without incomplete information including age at diagnosis, marital status, race, pathological extension, regional lymphonode

Patients with incomplete follow-up infomation

Surgery not performed

\section{Eligible patients}

$(\mathrm{n}=95,812)$

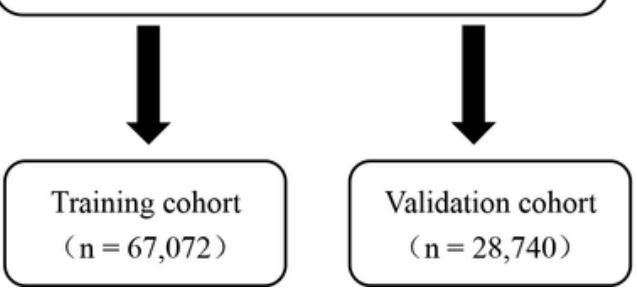

\section{Figure 1}

Flowchart describing the inclusion and exclusion criteria of patients in the Surveillance, Epidemiology, and End Results (SEER) database, 2004-2016. 
A

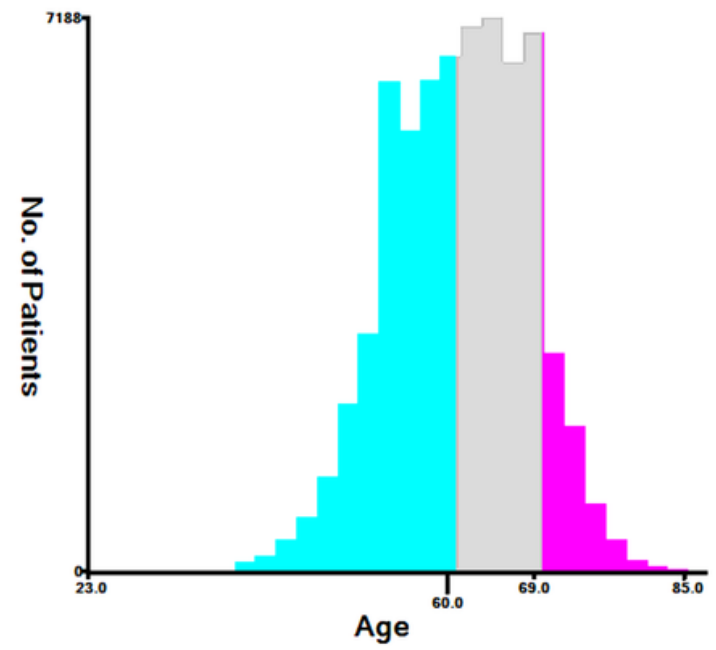

C

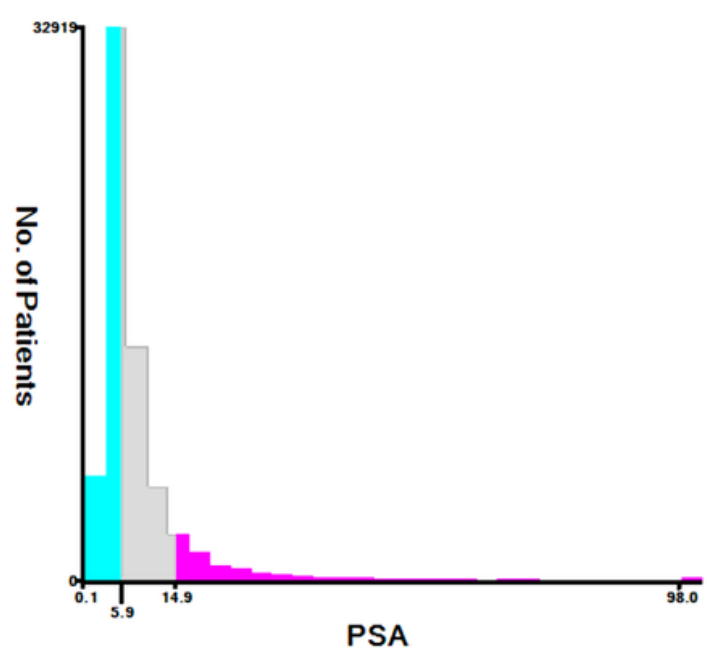

B

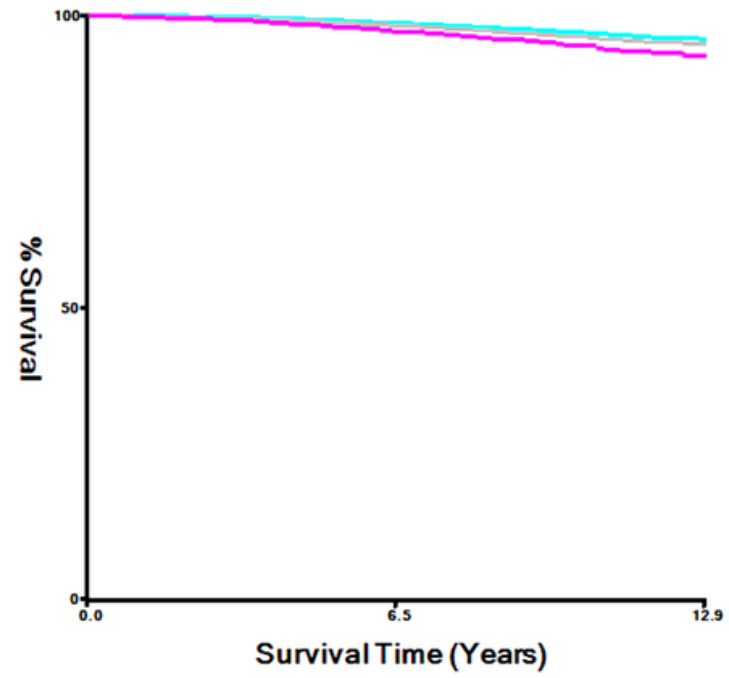

D

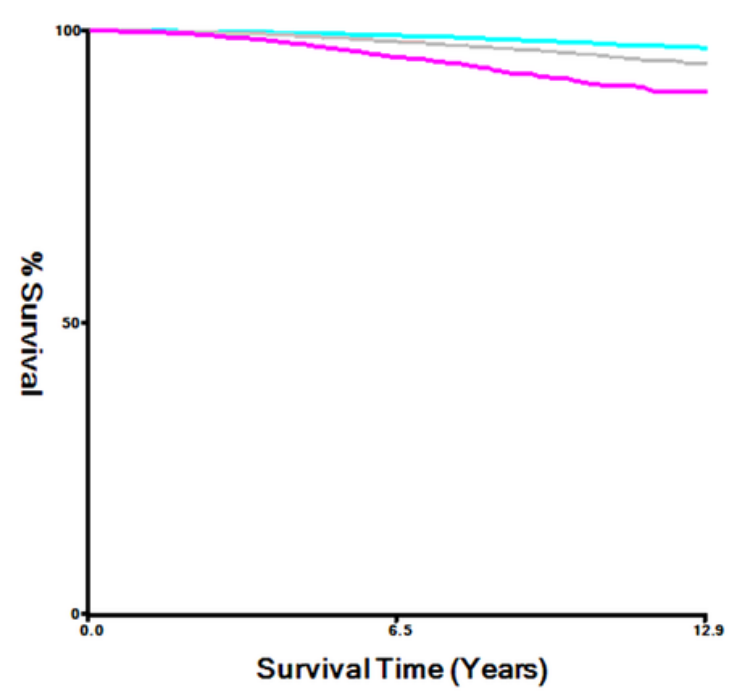

Figure 2

Determination of the optimal cut-off values of age at diagnose (A, B), and prostate specific antigen (PSA) level (C, D). The Optimal cut-off values were identified by the X-tile software according the difference of cancer-specific survival outcomes. 
Points

Age

Race

Marital_status

PSA

GS

Pathological_extension

Regional_nodes

Total Points

5-year cancer specific mortality

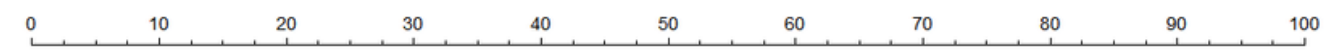

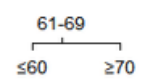
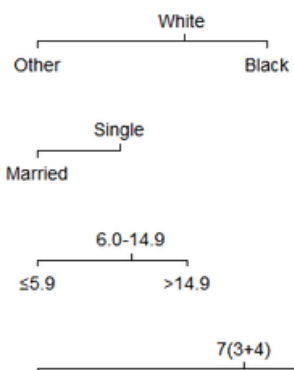

$7(3+4)$

$7(4+3)$

Adjacent-structures-invasion

Extracapsule-invasion Adjacent-structures

positive

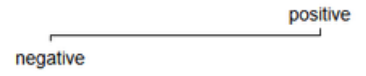

60
80

100

120

$\begin{array}{llll}160 & 180 & 200 & 220\end{array}$

$\begin{array}{llllll}0.05 & 0.1 & 0.15 & 0.2 & 0.25 & 0.30 .350 .4\end{array}$

\section{Figure 3}

Nomogram for predicting the cancer specific mortality (CSM) at 5 years in prostate cancer patients undergoing radical prostatectomy. Note: 1.Black: African American, White: Caucasian, Other: American Indian/AK Native, Asian/Pacific Islander 2.Single: Divorced, Separated, Single (never married), Widowed, unmarried

\section{A}

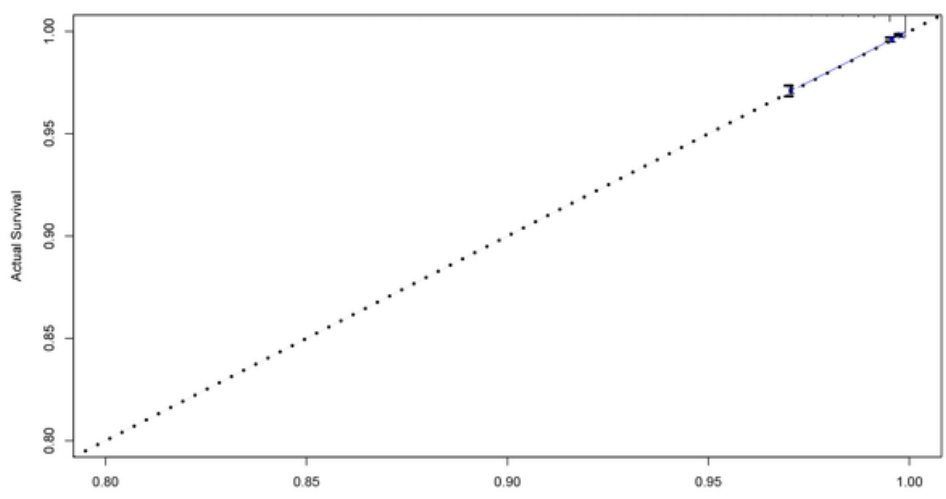

B

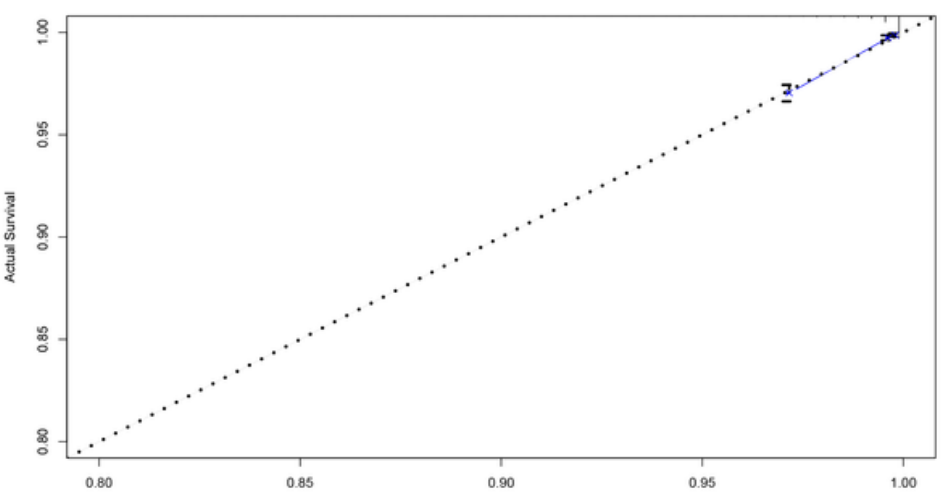

Figure 4 
Calibration curves for comparing the degree of agreement between the actual survival outcome and the survival outcome predicted by the nomogram in the training cohort $(A)$ and the validation cohort $(B)$. The horizontal axis is the survival rate predicted by the nomogram, and the vertical axis is the actual survival rate. The dashed line indicates the predicting survival rate completely fits the actual survival rate.

A

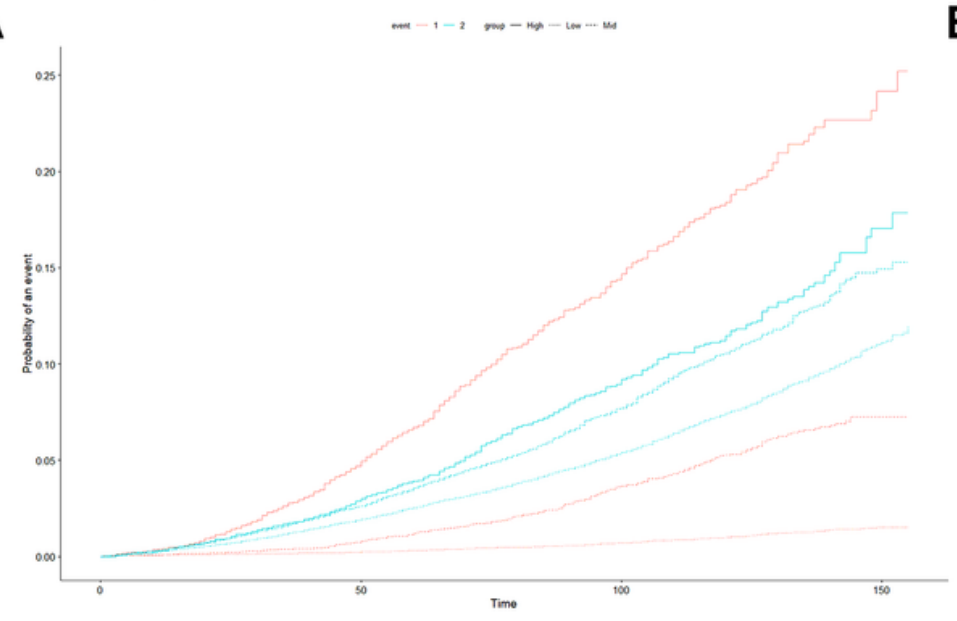

C

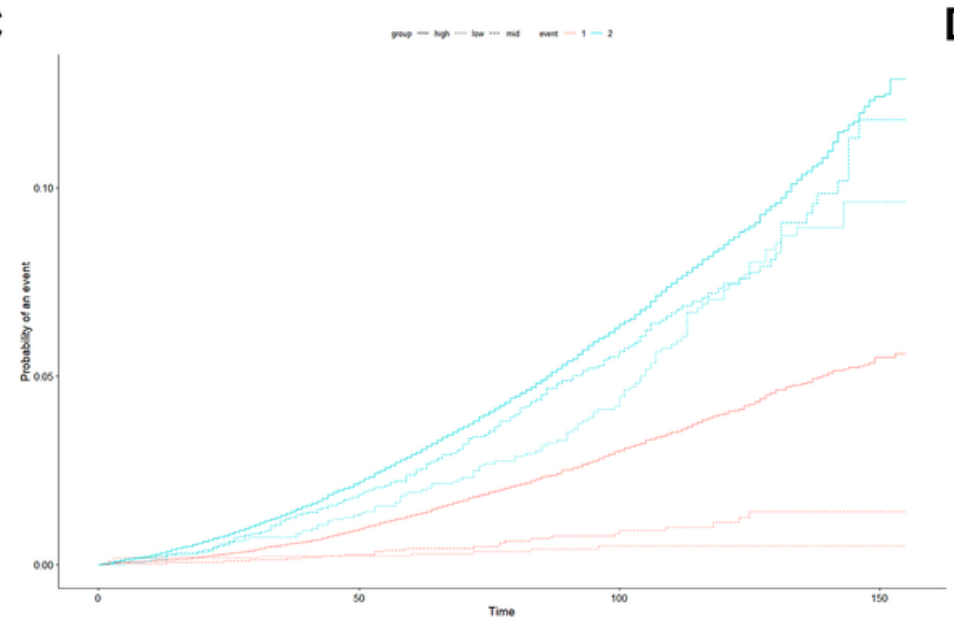

B

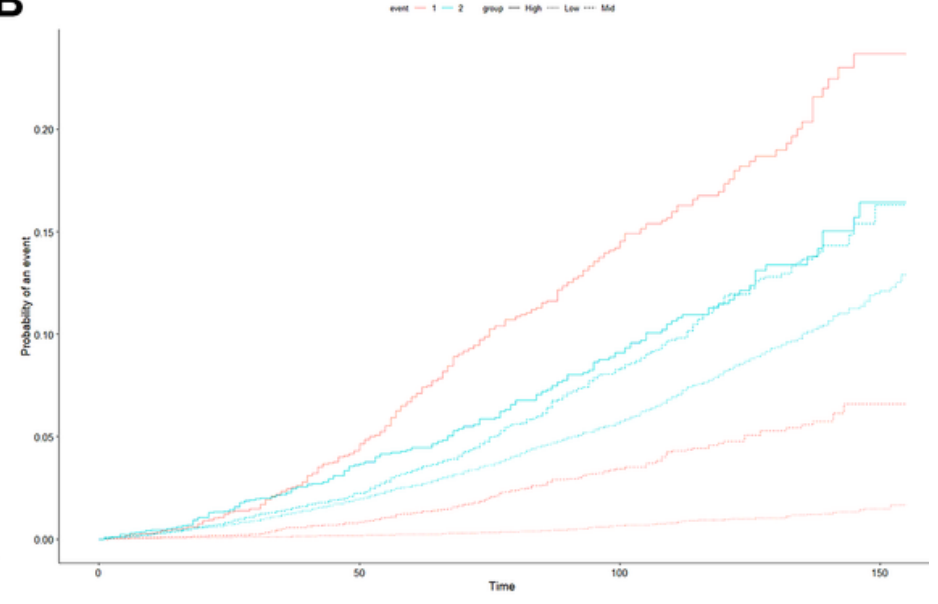

D

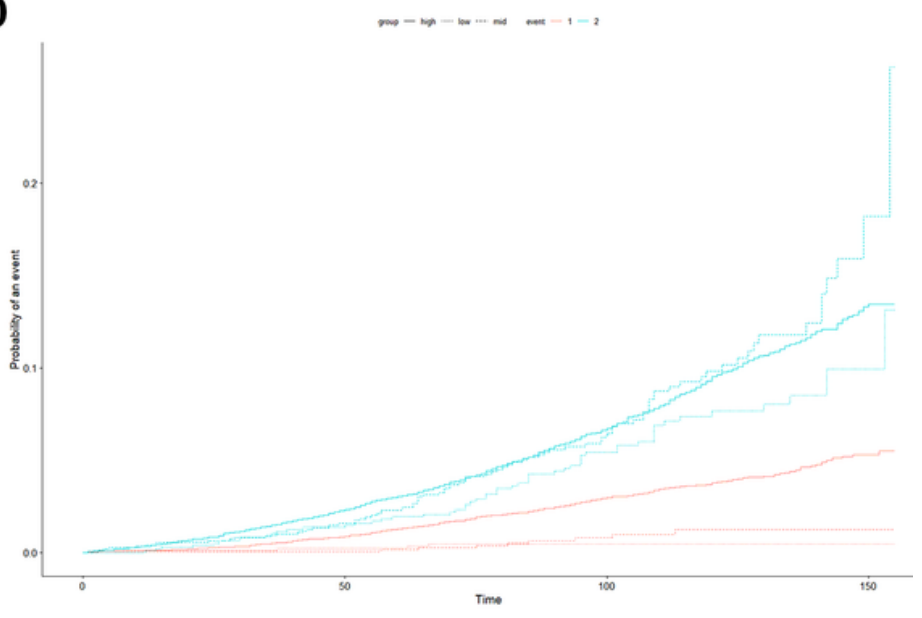

\section{Figure 5}

Cumulative incidence function (CIF) curves of different risk stratification systems for cancer specific mortality (CSM) in prostate cancer patients undergoing surgery. Nomogram risk stratification and EAU risk stratification based on D' Amico risk stratification were tested in the training cohort $(A, C)$ and validation cohort $(B, D)$. As event 1 , cancer-specific death was represented by red lines, and other causespecific death was represented by event 2 , and blue lines. 